\title{
Präferenzfreie Strategien zum Absichern von Wechselkursrisiken
}

\author{
Günter Franke * \\ Universität Konstanz
}

\section{Einleitung ${ }^{1}$}

In vielen Beiträgen wird die Politik von Unternehmen zum Absichern von Risiken untersucht. Da die Absicherungspolitik auf die Produktions- und Absatzpolitik zurückwirkt, werden oft beide Politiken gemeinsam untersucht. Prominentes Beispiel ist die Exportpolitik von Unternehmen bei Wechselkursrisiko. Frühe Arbeiten haben gezeigt, dass ein Unternehmen ohne Absicherung seine optimale Exportmenge reduziert, wenn der Wechselkurs riskant statt deterministisch ist. Die Begründung ist einfach: Bei Wechselkursrisiko wird der erwartete Gewinn aus einer marginalen Erhöhung der Exportmenge um eine Risikoprämie vermindert, so dass die optimale Exportmenge sinkt. Unter strengeren Annahmen an die Nutzenfunktion oder die Verteilungsfunktion des Wechselkurses folgt zudem, dass die optimale Exportmenge um so kleiner ist, je höher das Wechselkursrisiko ist.

Der Einfluss des Wechselkursrisikos auf die Exportpolitik verschwindet, wenn es einen vollkommenen Kapitalmarkt gibt, in dem alle Risiken abgesichert werden können. Dann gilt die Fisher-Separation: Jeder stochastische Zahlungsbetrag kann zu einem gegebenen Marktpreis veräußert werden. Die optimale Exportmenge ist erreicht, wenn die Grenzkosten mit dem Grenzertrag übereinstimmen, also dem Marktwert des stochastischen Exportgrenzerlöses. Dies bedeutet nicht, dass das Unternehmen keinerlei Wechselkursrisiken übernimmt. Wenn es eine Risikoprämie für die Übernahme von Wechselkursrisiken gibt, dann lohnt es sich aus spekulativen Gründen, ein Wechselkursrisiko zu übernehmen.

Die Wirklichkeit liegt zwischen der perfekten Absicherbarkeit und der Nicht-Absicherbarkeit von Risiken. Häufig existieren Terminkontrakte auf Wechselkursrisiken mit einer Laufzeit bis zu zwei Jahren, ebenso Optionskontrakte und Währungsswaps. Eine perfekte Absicherbarkeit von Wechselkursrisiken ist damit allerdings nicht gegeben. Folglich sind Export- und Absicherungsentscheidung nicht trennbar und müssen gemeinsam optimiert werden.

Mag die gemeinsame Optimierung von Export und Absicherung schon im Einperioden-Modell schwierig sein, so ist sie es erst recht im Mehrperioden-Modell. Die optimale Exportpolitik in einer Periode mag abhängig sein von der in benachbarten Perioden. Dies ist zu erwarten, wenn der Wechselkurs nicht einem einfachen Prozess gehorcht und/oder die Risikopräferenz des Unternehmens nicht durch konstante absolute oder relative Risikoaversion gekennzeichnet ist. Dann hängt die optimale Politik in einer Periode nicht nur von der Verteilung des Wechselkurses am Ende der Periode ab, sondern auch von der optimalen Nutzung des Entscheidungsspielraumes in zukünftigen Perioden, die ihrerseits von den Verteilungen des Wechselkurses in diesen Perioden abhängt.

\footnotetext{
${ }^{\star}$ Für wertvolle Verbesserungshinweise danke ich Axel Adam-Müller und Jeanette Roth.

${ }^{1}$ Jochen Wilhelm hat sich intensiv mit der intertemporalen Finanzmarkttheorie auseinandergesetzt, die auch in diesem Beitrag eine zentrale Rolle spielt. Zu erwähnen sind u.a. sein Beitrag (1992) zu Fristigkeitsstruktur und Zinsänderungsrisiko, in dem er das intertemporale Bond-Portfolio-Management mit der Zinsstrukturtheorie verknüpft. In einem anderen Beitrag (1999) veranschaulicht er das Ho-Lee Modell zur Zinsstruktur anschaulich anhand von intuitiv leicht nachvollziehbaren stochastischen Diskontfaktoren.
} 
Mit anderen Worten, die optimale Politik in einer Periode hängt nicht nur von der Verteilung des Wechselkurses in dieser Periode ab, sondern auch vom zukünftigen Entscheidungsspielraum. Insbesondere dient die Absicherungspolitik in einer Periode nicht nur der Absicherung gegen das Wechselkursrisiko dieser Periode, sondern auch der Absicherung gegen stochastische Änderungen des zukünftigen Entscheidungsspielraums. In einer solchen Welt kann eine optimale Politik nicht von Periode zu Periode abgeleitet werden, vielmehr ist eine optimale Strategie über alle Perioden gleichzeitig abzuleiten. Dies ist eine komplizierte Aufgabe. Sie erfordert ein präzises Verständnis der Prozesse, denen die relevanten Risikofaktoren unterworfen sind, sowie der Dynamik sequentieller Entscheidungen. Daher stellt sich die Frage, ob es nicht Situationen gibt, in denen die optimale Politik einfachen Regeln folgt, so dass komplizierte Modelle überflüssig sind.

Zweck dieses Beitrags ist es, mehrperiodige Modelle für ein Exportunternehmen zu untersuchen, in denen sich eine einfache Export- und Absicherungspolitik als optimal erweist. Eine Politik ist besonders einfach, wenn sie myopisch ist, wenn sie also nur von der Wechselkursverteilung der jeweiligen Periode abhängt und folglich keine Rücksicht auf das zukünftige Entscheidungsfeld zu nehmen braucht. "Einfache" Politiken machen indessen nur Sinn, wenn sie auf plausiblen Annahmen beruhen.

Besonders problematisch ist die Vorgabe einer "Nutzenfunktion des Unternehmens", um die Risikoaversion der Entscheidungsträger zum Ausdruck zu bringen. Nicht nur haben diese unterschiedliche Risikopräferenzen, sondern auch die konkrete Abbildung in einer Nutzenfunktion wirft Probleme auf. Daher soll in diesem Beitrag untersucht werden, unter welchen Voraussetzungen die optimale Export- und Absicherungspolitik von der gewählten Nutzenfunktion unabhängig ist. Es leuchtet unmittelbar ein, dass diese Präferenzfreiheit nur bestehen kann, wenn es keine Risikoprämie im Devisenmarkt gibt. Gäbe es eine solche, dann würde ein sehr risikoaverses Unternehmen keine spekulative Position im Devisenmarkt eingehen, im Gegensatz zu einem wenig risikoaversen Unternehmen. Zwar gibt es auch im Devisenmarkt kleine positive oder negative Risikoprämien. Jedoch ist in einem freien Devisenmarkt, der nicht durch staatliche Intervention oder Regulierung verzerrt ist, mit einer Risikoprämie nahe bei 0 zu rechnen. Die Annahme einer Risikoprämie von 0 erscheint daher gerechtfertigt. Um die Analyse einfach zu gestalten, wird unterstellt, dass es neben dem Wechselkurs keine Risikofaktoren gibt, die das Unternehmen zu beachten hat. Dies ist zweifellos eine restriktive Annahme. Sie schließt auch Inflationsrisiken aus. Deterministische Inflationsraten werden durch eine entsprechende Anpassung der risikofreien Nominalzinssätze ausgeglichen. Das Modell kann daher vereinfacht werden, indem alle Parameter auf realer statt nominaler Basis definiert werden.

Eine zentrale, aber durchaus plausible Annahme des Beitrags wird sein, dass der reale Wechselkurs einem Ornstein-Uhlenbeck-Prozess gehorcht. Das Unternehmen verkauft im fremden Markt unter den Bedingungen monopolistischen Wettbewerbs. Das bedeutet, dass der Exportgewinn eine ansteigende, streng konvexe Funktion des Wechselkurses ist. Das Unternehmen kann lediglich kurzfristige Devisenterminkontrakte zur Absicherung des Wechselkursrisikos einsetzen, so dass eine perfekte Absicherung nicht möglich ist. Dennoch erweist sich, unabhängig von der Nutzenfunktion, eine einfache deltaneutrale Absicherungspolitik als optimal. Bei dieser Politik verkauft das Unternehmen in jeder Periode Devisenterminkontrakte, so dass der Unternehmensgewinn aus Export und Absicherung vom Wechselkurs dann lokal unabhängig ist, wenn der Wechselkurs dem erwarteten Wechselkurs gleicht.

Die Ergebnisse erlauben auch, den Einfluss der Wechselkursvolatilität auf den Unternehmenswert zu kennzeichnen. Zwei Hypothesen stehen sich gegenüber. Die traditionelle Risikoaversionshypothese besagt, dass eine Zunahme der Volatilität die Exporte dämpft und den Wert des exportierenden Unternehmens senkt. Die Exportoptionshypothese geht davon aus, dass der Export eine Realoption des Unternehmens ist. Der Wert dieser Option steigt mit der Volatilität des Wechselkurses. Folglich wächst der Wert des exportierenden Unternehmens mit der Wechselkursvolatilität. Beide Hypothesen widersprechen sich. Während die Risikoaversionshypothese auf Risikoaversion und nicht absicherbaren Wechselkursrisiken beruht, stellt die Exportoptionshypothese auf die Flexibilität des Unternehmens ab, seine Exportmenge dem Wechselkurs anzupassen. Wenn der Wechselkurs steigt (fällt), erhöht (senkt) das Unternehmen seine Exportmenge und er- 
zielt dadurch einen Anpassungsgewinn. Je stärker der Wechselkurs variiert, um so höher ist der erwartete Anpassungsgewinn, um so höher ist der Wert des exportierenden Unternehmens.

Beide Hypothesen sind indessen problematisch. Die Risikoaversionshypothese vernachlässigt die Realoption; die Optionshypothese unterstellt einen vollkommenen und vollständigen Kapitalmarkt, auf dem alle Risiken abgesichert werden können. Dieser Beitrag soll daher auch zur Klärung des Geltungsbereichs dieser Hypothesen beitragen.

Der Beitrag ist wie folgt aufgebaut. Im 2. Abschnitt wird kurz auf die relevante Literatur eingegangen. Der 3. Abschnitt kennzeichnet die Modellsituation. Im 4. Abschnitt werden zunächst notwendige und hinreichende Bedingungen für eine präferenzfreie Politik angegeben. Dann wird die optimale präferenzfreie Export- und Absicherungspolitik abgeleitet. Im 5. Abschnitt werden die Ergebnisse diskutiert und ein Resümee gezogen.

\section{Literaturskizze}

Es gibt eine sehr umfangreiche Literatur zum Absichern von Wechselkursrisiken in Einperiodenmodellen, z.B. Holthausen (1979), Benninga, Eldor und Zilcha (1985), Kawai und Zilcha (1986), Ware und Winter (1988), Broll und Wahl (1992), Adam-Müller (1995), Breuer (2000). In diesen Beiträgen werden zahlreiche Fragen angesprochen, unter anderem die Frage, unter welchen Bedingungen Export- und Absicherungsentscheidungen trennbar sind. Separation existiert immer dann, wenn alle Exportrisiken vollständig abgesichert werden können. Dann tritt an die Stelle des stochastischen Exporterlöses der deterministische Marktwert des Erlöses. Sind die Exportrisiken nicht vollständig absicherbar, dann sind Export- und Absicherungsentscheidung untrennbar.Gollier (1995) untersucht in einem allgemeinen Entscheidungsmodell, welche Änderungen der Verteilungsfunktion des exogenen Preisrisikos den risikoscheuen Entscheidungsträger veranlassen, seine risikobehaftete Aktivität einzuschränken. Adam-Müller (2002) analysiert die Export- und Absicherungsentscheidung unter Inflationsrisiko. Broll, Wahl und Zilcha (1999) erweitern diese Modelle auf den Mehrperiodenfall und prüfen die Bedingungen für Separation und full hedge.Neuberger (1999) untersucht revolvierende Strategien, um ein langfristiges Risiko durch Einsatz kurzfristiger Terminkontrakte abzusichern. In anderen Mehrperiodenmodellen wird Export als Realoption betrachtet. Die optimale Ausübung dieser Option analysieren Franke (1991), Sercu und van Hulle (1992) sowie Mello, Parsons und Triantis (1995). Franke (1991) zeigt, dass der Wert der Exportoption mit der Wechselkursvolatiliät wachst. Mello et al (1995) untersuchen eine Firma, die ihre

Beschaffungs- und Absicherungspolitik flexibel gestaltet. Der Unternehmenserfolg hängt ab von den Fähigkeiten des Unternehmens, Absicherungsstrategie und Beschaffungsflexibilität aufeinander abzustimmen.

In einem allgemein gehaltenen Modell untersuchen Froot, Scharfstein und Stein (1993) Risikomanagement als einen Mechanismus, Investitions- und Finanzierungsentscheidungen zu koordinieren. In ihrem Modell profitiert eine risikoneutrale Firma von einer Absicherungspolitik, wenn die Grenzrendite von Investitionen sinkt und das optimale Investitionsvolumen mit dem Unternehmensvermögen wächst. Franke (2003) wendet eine ähnliche Idee auf internationale Investitionsund Absicherungsstrategien an. Ungern-Sternberg und Weizsäcker (1990) ergänzen die Absicherungsentscheidung um strategische Aspekte.

Inzwischen gibt es auch eine umfangreiche empirische Literatur zum Absichern von Exportrisiken. Alayannis und Ofek (2001) finden, dass Unternehmen Wechselkursderivate zum Absichern, nicht zum Spekulieren nutzen. Haushalter (2000) untersucht das Absicherungsverhalten von Ölund Gasfirmen. Er beobachtet, dass höher verschuldete Unternehmen stärker absichern. Auch wird die Absicherungspolitik durch Economies of scale-Effekte und durch Basisrisiken beeinflusst. Adam (2002) untersucht, inwieweit Goldminenbetreiber ihr Preisrisiko absichern. Er beobachtet, dass im Durchschnitt die Firmen 28 Prozent ihrer erwarteten Produktion im ersten Jahr absichern, 15 Prozent im zweiten und 7 Prozent im dritten Jahr. Guay und Kothari (2003) finden, dass Unternehmen Finanzderivate nur in sehr bescheidenem Umfang zum Absichern von Risiken einsetzen. 
In einem theoretisch-empirischen Beitrag untersuchen Bodnar, Dumas und Marston (2002) die subtilere Frage, wie Unternehmen die Fremdwährungspreise ihrer Exportprodukte Wechselkursänderungen anpassen und wie dies ihr Wechselkursrisiko beeinflusst. Damit werden auch Wettbewerbsaspekte einbezogen.

Zahlreiche Papiere untersuchen Wechselkursprozesse. Einen Überblick bietetEngle (1996). Wie die Forward-Anomalie zeigt, können Risikoprämien im Devisenmarkt bestehen. Jedoch erscheinen diese gering und wechseln das Vorzeichen. Auch gibt es starke empirische Evidenz für Kaufkraftparität auf lange Sicht. Dies deutet auf mean-reversion in den realen Wechselkursen, die über einen Ornstein-Uhlenbeck Prozeß modelliert werden kann.

\section{Das Entscheidungsmodell}

Untersucht wird die Politik einer Firma, die ein Produkt in den Perioden1, 2, ,..,T exportiert. Der Wechselkurs ist stochastisch. In jeder Periode $t$ entscheidet die Firma zu Beginn der Periode, also im Zeitpunkt $(t-1)$, über die Exportmenge. Sie bekommt den Exporterlös in Fremdwährung am Periodenende, also im Zeitpunkt $t$. Der Erlös wird dann zum Wechselkurs $e_{t}$ in Heimatwährung getauscht. Der Wechselkurs ist definiert als Zahl der Einheiten der Heimatwährung pro Fremdwährungseinheit. Die Firma entscheidet über die Exportmenge, bevor sie den Wechselkurse $e_{t}$ kennt. Sie kennt zu diesem Zeitpunkt den Wechselkurs $e_{t-1}$.

Der Exportmarkt ist durch monopolistischen Wettbewerb [Dixit und Stiglitz (1977)] gekennzeichnet. Die Produkte verschiedener Firmen sind unvollkommene Substitute. Die Nachfragekurve für das Produkt der Firma ist fallend, ausgehend von einem gegebenen Wechselkurs. Außerdem fällt der Preis, wenn die aggregierte Menge der von der Konkurrenz verkauften Produkte steigt. Ein Anstieg des Wechselkurses begünstigt die Firma wie auch ihre Konkurrenten, wenn sie ebenfalls im Heimatland domizilieren. Wenn die Konkurrenz ihr Exportvolumen erhöht, sinkt der Exporterlös der Firma. Es wird unterstellt, dass dieser indirekte, konkurrenzinduzierte negative Effekt einer Wechselkurserhöhung geringer ist als der direkte positive Effekt. Dies präzisiert Annahme 3.1. $X_{t}$ bezeichne die Exportmenge der Firma in Periode $t$.

\section{Annahme 3.1:}

a) Bei einer gegebenen Exportmenge $X_{t}$ der Firma steigt ihr Exporterlös in Heimatwährung mit dem Wechselkurs $e_{t-1}$.

b) Ein Anstieg des Wechselkurses $e_{t-1}$ veranlasst die Firma, ihre Exportmenge $X_{t}$ zu erhöhen.

c) Eine Konvexität des Exportergebnisses im Wechselkurs kann durch Konkurrenzeffekte nicht in Konkavität verändert werden.

Annahme 3.1 besagt, dass die positiven Effekte eines Wechselkursanstiegs durch die Konkurrenz gemindert, aber nicht aufgehoben werden können. Dies erscheint bei monopolistischem Wettbewerb realistisch. Die Firma entscheidet hier über $X_{t}$ im Zeitpunkt $(t-1)$, wenn sie den Wechselkurs $e_{t-1}$ kennt. Dasselbe gilt für die Konkurrenten. $R_{t}=R_{t}\left(X_{t}, e_{t-1}\right)$ sei der Exporterlös der Firma in Fremdwährung im Zeitpunkt $t$. Diese Funktion sei zweimal differenzierbar in $X_{t}$ und $e_{t-1}$. Wenn die Konkurrenten ihre Exportmenge mit dem Wechselkurs $e_{t-1}$ erhöhen, dann folgt $\partial R_{t}\left(X_{t}, e_{t-1}\right) / \partial e_{t-1}<0$. Wie üblich, sei der Exporterlös in Fremdwährung eine steigende, konkave Funktion der Exportmenge $X_{t}$. Zur Vereinfachung der Schreibweise sei $R_{t}^{\prime}\left(X_{t}, e_{t-1}\right) \equiv \partial R_{t}(\cdot) / \partial X_{t}>0$ und $R_{t}^{\prime \prime}\left(X_{t}, e_{t-1}\right) \equiv \partial^{2} R_{t}(\cdot) / \partial X_{t}^{2}<0$.

$C_{t}\left(X_{t}\right)$ sei die Kostenfunktion der Firma, wobei alle Kosten auszahlungswirksam seien. Sie ist in Heimatwährung angegeben. Die im Exportland anfallenden Kosten werden vom Fremdwährungserlös abgezogen. Die Kostenfunktion ist steigend und zweimal differenzierbar. Ist sie nicht konvex, dann sei ihre Konkavität genügend gering, um ein inneres Optimum für die Exportmenge zu gewährleisten. Es gebe keine Übertragungseffekte auf andere Perioden, d.h., Erlöse und Kosten in Periode $t$ sind unabhängig vom Geschehen in benachbarten Perioden. Kosten und Wechselkurse sind in realen Kaufkrafteinheiten des Heimatlandes definiert. 
Der reale Wechselkurs folge einem additiven Ornstein-Uhlenbeck-Prozess mit den Innovationen $\varepsilon_{t}$,

$$
e_{t}=\gamma e_{t-1}+(1-\gamma) \bar{e}+\varepsilon_{t} ; \quad \gamma \in(0,1) ; \quad t=1, \ldots, T-1 .
$$

$\bar{e}$ ist der langfristige Gleichgewichts-Wechselkurs, $\gamma$ die mean reversion-Geschwindigkeit und $\varepsilon_{t}$ die stochastische Komponente der Wechselkursänderung, die (a) unabhängig vone $e_{t-1}$ und $\varepsilon_{t-1}$ sei und (b) einen Erwartungswert von 0 habe. $\varepsilon_{t}$ bezeichnet die Wechselkursinnovation. $\gamma$ und der Definitionsbereich von $\varepsilon_{t}$ sind so gewählt, dass negative Wechselkurse ausgeschlossen sind.

Die Firma kann einperiodige Terminkontrakte auf den Wechselkurs handeln. Im Devisenmarkt gebe es weder eine Risikoprämie noch Transaktionskosten. Folglich gilt für den Terminkurs $f_{t-1}$ im Zeitpunkt $(t-1)$

$$
f_{t-1}=E_{t-1}\left(e_{t}\right)=\gamma e_{t-1}+(1-\gamma) \bar{e} ; \quad t=1, \ldots, T .
$$

Die Firma kann Geld zum Einheitszinssatz risikofrei anlegen und aufnehmen. Dieser Zinssatz sei im Zeitablauf konstant. Die Firma maximiert stets ihren erwarteten Nutzen anhand einer zeitadditiven, konkaven Nutzenfunktion.

\section{Die optimale Politik}

In diesem Abschnitt wird zunächst ein Lemma präsentiert, das in allgemeiner Form eine notwendige und hinreichende Bedingung für eine präferenzfreie Absicherungspolitik angibt. Sodann wird die optimale Exportpolitik abgeleitet. Es wird gezeigt, daß das voll abgesicherte Exportergebnis eine steigende, konvexe Funktion des Wechselkurses ist. Unter bestimmten Bedingungen ist diese Konvexität konstant. Für diesen Fall wird sodann die optimale dynamische Absicherungspolitik abgeleitet und anhand einer Graphik veranschaulicht.

\subsection{Bedingungen für eine präferenzfreie Politik}

Da es sehr schwierig ist, eine "Nutzenfunktion der Firma" anzugeben, werden in diesem Teilabschnitt Bedingungen für eine präferenzfreie Politik abgeleitet.

Die Firma optimiert ihre Export- und Absicherungspolitik. Bezüglich der Exportpolitik ist Präferenzfreiheit immer dann gegeben, wenn alle Exportrisiken perfekt abgesichert werden können und daher Separation gilt. Weder Präferenzen noch Wahrscheinlichkeitsurteile spielen dann eine Rolle für die Exportentscheidung. Diese Situation ist hier gegeben, wie im nächsten Teilabschnitt verdeutlicht wird.

Schwieriger ist die Frage zu beantworten, unter welchen Bedingungen die optimale Absicherungspolitik präferenzfrei ist. Eine notwendige Bedingung wurde bereits genannt, nämlich dass die Risikoprämie im Devisenmarkt gleich 0 ist. Sonst würde, ceteris paribus, das spekulative Engagement im Devisenmarkt um so größer, je weniger risikoscheu die Firma ist. Sei $u_{t}\left(\pi_{t}\right)$ eine beliebige konkave Nutzenfunktion im Zeitpunkt $t$. Der Gewinn des Unternehmens $\pi_{t}$ setzt sich zusammen aus dem Exportergebnis und dem Absicherungsergebnis. Wenn das Unternehmen $Z_{t-1}$ Terminkontrakte im Zeitpunkt $(t-1)$ verkauft, dann erzielt es daraus im Zeitpunkt $t$ ein Absicherungsergebnis

$$
Z_{t-1}\left(f_{t-1}-e_{t}\right)=-Z_{t-1} \varepsilon_{t} .
$$

Die Bedingung für eine optimale Zahl von zu verkaufenden Kontrakten lautet dann, ausgehend von der Nutzenfunktion $u_{t}$ 


$$
E_{t-1}\left[u_{t}^{\prime}\left(\pi_{t}\right)\left(-\varepsilon_{t}\right)\right]=0
$$

oder

$$
\operatorname{cov}_{t-1}\left(u_{t}^{\prime}\left(\pi_{t}\right), \varepsilon_{t}\right)=0,
$$

wobei $u_{t}^{\prime}\left(\pi_{t}\right)$ den Grenznutzen des Ergebnisses $\pi_{t}$ bezeichnet. Ist das Exportergebnis deterministisch, dann ist offenbar $Z_{t-1}=0$. Denn dann ist das Gesamtergebnis $\pi_{t}$ deterministisch und somit Gleichung (5) erfüllt. Da das Optimierungsproblem konvex ist, ist das Optimum eindeutig.

Ist indessen (im Mehrperiodenmodell) das Exportergebnis stochastisch, dann ist es in diesem einfachen Modell von dem einzigen Risikofaktor "Wechselkurs" abhängig. Es kommt dann darauf an, das Exportergebnis bestmöglich durch Terminkontrakte abzusichern. Dies ist perfekt möglich, wenn das Exportergebnis eine lineare Funktion des Wechselkurses ist. Denn das Absicherungsergebnis ist ebenfalls linear im Wechselkurs. Der interessante Fall ist deshalb der, in dem das Exportergebnis eine nichtlineare Funktion des Wechselkurses ist. Für diesen Fall gilt das folgende Lemma. Zugrunde liegt ein Gesamtergebnis der Firma

$$
\pi_{t}=\pi_{t}^{P}-Z_{t-1} \ni_{t},
$$

wobei $\pi_{t}^{P}$ das Exportergebnis ist, $Z_{t-1}$ die Zahl der von einem Finanzderivat zu verkaufenden Einheiten und $\ni_{t}$ das Ergebnis pro Einheit des Derivats. Ist für das Finanzderivat ein Preis im Zeitpunkt $(t-1)$ zu zahlen, so wird dieser risikofrei auf den Zeitpunkt $t$ aufgezinst und von $\ni_{t}$ abgezogen. In einem Markt ohne Risikoprämie gilt $E_{t-1}\left(\ni_{t}\right)=0$.

Lemma 4.1. Die optimale zu verkaufende Stückzahl des Finanzderivats ist präferenzfrei dann und nur dann, wenn bei optimaler Politik gilt

$$
E_{t-1}\left(\ni_{t} \mid \pi_{t}\left(\ni_{t}\right)=\pi_{t}\right)=0 \quad \forall \quad \pi_{t},
$$

wenn also dasselbe Gesamtergebnis $\pi_{t}$ bei unterschiedlichen Ergebnisrealisationen $\ni_{t}$ erzielt wird, die sich im Erwartungswert gerade ausgleichen, $\forall \pi_{t}$.

Beweis. Zunächst wird gezeigt, dass Bedingung (6) hinreichend für Präferenzfreiheit von $Z_{t-1}$ ist.

Die zu Bedingung (4) analoge Bedingung für den optimalen Einsatz des Finanzderivats kann umgeschrieben werden zu

$$
E_{\pi, t-1}\left[E_{\ni, t-1}\left\{\ni_{t} \mid \pi_{t}\left(\ni_{t}\right)=\pi_{t}\right\} u_{t}^{\prime}\left(\pi_{t}\right)\right]=0 .
$$

In Gleichung (7) wird zunächst bei gegebenem $\pi_{t}$ der Erwartungswert über $\ni_{t}$ und sodann der über $\pi_{t-1}$ gebildet.

Betrachte für jedes mögliche Ergebnis $\pi_{t}$ die Werte von $\ni_{t}$, die dieses Ergebnis $\pi_{t}$ bei einer gegebenen Politik erzeugen. Wenn der bedingte Erwartungswert dieser $\ni_{t}{ }^{-}$Werte gleich 0 ist, dann wird $u_{t}^{\prime}\left(\pi_{t}\right)$ jeweils mit 0 multipliziert. Folglich ist Gleichung (7) erfüllt.

Jetzt ist die Notwendigkeit von Bedingung (6) zu zeigen. Es gebe ein Ergebnisniveau $\pi_{t}=\pi_{t}^{0}$, für das Bedingung (6) nicht gilt. Gegeben sei eine optimale Politik $Z_{t-1}$, ausgehend von einer gegebenen Nutzenfunktion. Gleichung (4) gilt dann analog für das Derivat. Nun werde die Nutzenfunktion $u_{t}\left(\pi_{t}\right)$ an der Stelle $\pi_{t}^{0}$ und in ihrer unmittelbaren Nachbarschaft marginal verändert. Dann ist Bedingung (4) verletzt. Folglich ist die Absicherungspolitik nicht präferenzfrei. Also ist Bedingung (6) notwendig für Präferenzfreiheit der Absicherungspolitil

Die Bedingung (6) für eine präferenzfreie Absicherungspolitik ist recht streng. Um sie zu veranschaulichen, unterscheiden wir positive Werte von $\ni_{t}, \ni_{t}^{+}$, und negative Werte von $\ni_{t}, \ni_{t}^{-}$. Dann besagt Bedingung (6), dass der Erwartungswert der positiven Werte $\ni_{t}^{+}$, die zum optimalen Ergebnis $\pi_{t}^{0}$ führen, übereinstimmen muss mit dem absolut genommenen Erwartungswert der negativen Werte $\ni_{t}^{-}$, die ebenfalls zum Ergebnis $\pi_{t}^{0}$ führen. Dies muss für jedes Ergebnisniveau $\pi_{t}^{0}$ zutreffen. Diese Bedingung ist z.B. erfüllt, wenn $\ni_{t}$ symmetrisch um 0 verteilt ist und das Ergebnis $\pi_{t}\left(+\varepsilon_{t}\right)$ mit dem Ergebnis $\pi_{t}\left(-\varepsilon_{t}\right)$ übereinstimmt; $\forall \varepsilon_{t}$. Dieser Spezialfall wird später näher analysiert. 


\subsection{Die optimale Exportpolitik}

Im folgenden wird zuerst die optimale Exportpolitik, dann die optimale Absicherungspolitik abgeleitet.

Die optimale Exportpolitik ist leicht zu charakterisieren, wenn Separation besteht. Dies ist hier der Fall, weil es außer dem Wechselkurs keinen Risikofaktor gibt. Im Zeitpunkt $(t-1)$ entscheidet die Firma über die Exportmenge $X_{t}$, die im Zeitpunkt $t$ den Fremdwährungserlös $R_{t}\left(X_{t}, e_{t-1}\right)$ abwirft. Da im Zeitpunkt $(t-1)$ der einperiodige Devisen-Terminkurs gleich $f_{t-1}$ ist, ergibt sich ein abgesicherter, determinischer Erlös in Heimatwährung von $R_{t}\left(X_{t}, e_{t-1}\right) f_{t-1}$. Dann ergibt sich die optimale Exportmenge aus der klassischen Bedingung "Grenzkosten = Grenzerlös":

$$
R_{t}^{\prime}\left(X_{t}^{*} ; e_{t-1}\right) f_{t-1}=C_{t}^{\prime}\left(X_{t}^{*}\right) .
$$

Hinreichend für ein Gewinnmaximum ist

$$
R_{t}^{\prime \prime}\left(X_{t}^{*} ; e_{t-1}\right) f_{t-1}<C_{t}^{\prime \prime}\left(X_{t}^{*}\right) .
$$

Das voll abgesicherte Exportergebnis ist daher

$$
\pi^{p}\left(e_{t-1}\right)=R_{t}\left(X_{t}^{*} ; e_{t-1}\right) f_{t-1}-C_{t}\left(X_{t}^{*}\right),
$$

wobei $X_{t}^{*}$ von $e_{t-1}$ abhängt. Proposition 4.1 verdeutlicht den Zusammenhang zwischen dem Exportergebnis und dem Wechselkurs $e_{t-1}$.

Proposition 4.1. Das voll abgesicherte Exportergebnis $\pi^{P}\left(e_{t-1}\right)$ ist eine ansteigende, konvexe Funktion.

Beweis. Es gilt

$$
\frac{\mathrm{d} \pi^{P}}{\mathrm{~d} e_{t-1}}=\left[R_{t}^{\prime}\left(X_{t}^{*}, e_{t-1}\right) f_{t-1}-C_{t}^{\prime}\left(X_{t}^{*}\right)\right] \frac{\partial X_{t}^{*}}{\partial e_{t-1}}+\frac{\partial\left[R_{t}\left(X_{t}^{*}, e_{t-1}\right) f_{t-1}\right]}{\partial e_{t-1}}
$$

Der erste Ausdruck auf der rechten Seite von (10) ist wegen (8) gleich0, der zweite Ausdruck ist gemäß Annahme 3.1 a) positiv. Folglich ist $\delta \pi^{P} / \delta e_{t-1}>0$.

Die Konvexität des Exportergebnisses ist

$$
\frac{\mathrm{d}^{2} \pi^{P}}{\mathrm{~d} e_{t-1}^{2}}=\frac{\partial^{2}\left[R_{t}\left(X_{t}^{*}, e_{t-1}\right) f_{t-1}\right]}{\partial e_{t-1}^{2}}+\frac{\partial\left[R_{t}^{\prime}\left(X_{t}^{*}, e_{t}\right) f_{t-1}\right]}{\partial e_{t-1}} \frac{\partial X_{t}^{*}}{\partial e_{t-1}} .
$$

Der zweite Ausdruck auf der rechten Seite ist positiv. Dies folgt aus (8) und Annahme $3.1 \mathrm{~b}$ ). Differenziert man (8) nach $e_{t-1}$, so folgt

$$
\operatorname{sgn} \frac{\partial X_{t}^{*}}{\partial e_{t-1}}=\operatorname{sgn} \frac{\partial\left[R_{t}^{\prime}\left(X_{t}^{*}, e_{t}\right) f_{t-1}\right]}{\partial e_{t-1}} .
$$

Gemäß Annahme $3.1 \mathrm{~b})$ ist $\delta X_{t}^{*} / \delta e_{t-1}>0$, so dass der zweite Ausdruck positiv ist. Er dominiert gemäß Annahme $3.1 \mathrm{c}$ ) den ersten Ausdruck

Die Konvexität des voll abgesicherten Exportergebnisses folgt aus der Realoption der Firma, ihre Exportmenge dem Wechselkurs anzupassen. Bei gegebener Exportmenge und Fehlen der Konkurrenz würde das Exportergebnis linear mit dem Wechselkurs steigen. Durch die Anpassung der Exportmenge kann die Firma das Exportergebnis steigern; dies äußert sich in der Konvexität des Exportergebnisses im Wechselkurs.

Wie später gezeigt wird, vereinfacht sich die optimale Absicherungspolitik erheblich, wenn u.a. gilt, dass die Konvexität des Exportergebnisses im Wechselkurs konstant ist. Die Konvexität ist 
konstant, wenn $\partial \pi^{P} / \partial e_{t-1}$ linear in $e_{t-1}$ ist. Gemäß Gleichung (10) trifft dies zu, wenn (beachte: $\left.\partial f_{t-1} / \partial e_{t-1}=\gamma\right)$

$$
\frac{\partial R_{t}}{\partial e_{t-1}} f_{t-1}+R_{t}\left(X_{t}^{*}, e_{t-1}\right) \gamma=a_{t}+b_{t} e_{t-1} ; \quad b_{t}>0 .
$$

Da $\partial R_{t} / \partial e_{t-1}=\gamma \partial R_{t} / \partial f_{t-1}$ ist, lässt sich diese Gleichung umschreiben zu

$$
R_{t}\left(X_{t}^{*}, e_{t-1}\right)\left(1-\theta_{t}\right) \gamma=a_{t}+b_{t} e_{t-1} .
$$

Hierin ist $\theta_{t} \equiv-\partial \ln R_{t} / \partial \ln f_{t-1}$ die positive Elastizität des Exporterlöses bezüglich des Terminkurses, erzeugt ausschließlich durch Konkurrenzeffekte. Gibt es keine Konkurrenz, dann ist $\theta_{t}=0$. Ansonsten ist $\theta_{t} \in(0 ; 1)$.

Es ist schwierig, genaue Aussagen zu den Konkurrenzeffekten zu machen [siehe auch Bodnar u.a. (2002)]. Eine vereinfachende Annahme besteht darin, $\theta_{t}$ als vom Wechselkurs unabhängig anzusehen. Dann wächst der Exporterlös $R_{t}\left(X_{t}^{*}, e_{t-1}\right)$ linear im Wechselkurs. Wenn also der Terminkurs um $1 \%$ steigt, sinkt der Exporterlös infolge der Konkurrenz um $\theta_{t} \%$. Die Exportmenge muss dann so stark wachsen, dass nicht nur diese Erlösminderung ausgeglichen wird, sondern darüberhinaus der Exporterlös um $b_{t} \Delta e_{t-1} / \gamma$ wächst. $\Delta e_{t-1}$ ist der zugehörige Anstieg des Wechselkurses $e_{t-1}$.

Bei konstanter Konvexität des Exportergebnisses und konstanter Elastizität $\theta_{t}$ muss der Exporterlös gemäß (12) linear im Wechselkurs steigen. Folglich muss der Exporterlös in Heimatwährung eine quadratische Funktion des Wechselkurses sein. Demnach kann das abgesicherte Exportergebnis nur dann konstante Konvexität in $e_{t-1}$ aufweisen, wenn auch die Kosten $C_{t}\left(X_{t}^{*}\left(e_{t-1}\right)\right)$ linear oder quadratisch im Wechselkurs sind. Die genannten Bedingungen sind auch hinreichend für konstante Konvexität des abgesicherten Exportergebnisses im Wechselkurs. Diese Ergebnisse fasst Proposition 4.2 zusammen.

Proposition 4.2. Die Elastizität des Fremdwährungserlöses der Firma in bezug auf den Wechselkurs, ausgelöst durch Konkurrenzeffekte, sei bei optimaler Exportpolitik vom Wechselkurs unabhängig. Das voll abgesicherte Exportergebnis weist in bezug auf den Wechselkurs konstante Konvexität dann und nur dann auf, wenn sowohl der voll abgesicherte Exporterlös in Heimatwährung als auch die Produktionskosten quadratische Funktionen im Wechselkurs sind

Proposition 4.2 enthält testbare Annahmen und Ergebnisse. Kritischer Überprüfung bedarf insbesondere die These, dass die Firma auch bei niedrigem Wechselkurs exportiert. Es mag sein, dass die Firma auch dann exportiert, weil sie in Erwartung zukünftig höherer Wechselkurse den Exportmarkt nicht aufgeben möchte. Dies ist insbesondere dann plausibel, wenn die Eintrits- und Austrittskosten des Exportmarktes vergleichsweise hoch sind oder/und der Wechselkurs rasch zum langfristigen Gleichgewicht zurück tendiert. Letztlich kann nur empirisch geklärt werden, ob das voll abgesicherte Exportergebnis konstante Konvexität im Wechselkurs aufweist. Immerhin mag dies eine vertretbare Approximation sein.

\subsection{Die optimale Absicherungspolitik}

Aufgrund des Separationstheorems wurde die optimale Exportpolitik in einfacher Weise bestimmt. Der Fremdwährungserlös des Zeitpunktes t wird im Zeitpunkt $(t-1)$ vollständig durch Devisenterminkontrakte gegen das Wechselkursrisiko abgesichert. Eine darüber hinausgehende spekulative Position in Terminkontrakten kommt nicht in Frage, weil im Devisenmarkt keine Risikoprämie verdient werden kann. Daher ist eine volle Absicherung im Zeitpunkt $(t-1)$ optimal.

Das so erzielte Exportergebnis $\pi^{P}$ hängt jedoch vom Wechselkurs $e_{t-1} \mathrm{ab}$, da die optimale Exportmenge $X_{t}$ und der Terminkurs $f_{t-1}$ hiervon abhängen. Im Zeitpunkt $(t-2)$ ist $e_{t-1}$ noch nicht bekannt. Folglich ist in diesem Zeitpunkt $\pi^{P}\left(e_{t-1}\right)$ stochastisch. Die Firma entscheidet daher im Zeitpunkt $(t-2)$ über ihre Absicherungspolitik. 
Verkauft die Firma im Zeitpunkt $(t-2) Z_{t-2}$ einperiodige Devisenterminkontrakte zum Terminkurs $f_{t-2}$, so resultiert daraus im Zeitpunkt $(t-1)$ ein Ergebnis von $Z_{t-2}\left(f_{t-2}-e_{t-1}\right)$. Wird dieses bis zum Zeitpunkt $t$ risikofrei angelegt, so ergibt sich im Zeitpunkt $t$ insgesamt ein Ergebnis $\pi_{t}$ von

$$
\pi_{t}=\pi^{p}\left(e_{t-1}\right)+Z_{t-2}\left(f_{t-2}-e_{t-1}\right) r_{t-1, t} .
$$

Hierin bezeichnet $r_{t-1, t}$ den risikofreien Aufzinsungsfaktor für Periode $t$. Die Bedingung für eine optimale Absicherung lautet dann

$$
E_{t-2}\left[u_{t}^{\prime}\left(\pi_{t}\right)\left(f_{t-2}-e_{t-1}\right)\right]=E_{t-2}\left[u_{t}^{\prime}\left(\pi_{t}\right)\left(-\varepsilon_{t-1}\right)\right]=0 .
$$

Gemäß Lemma 4.1 ist die optimale Absicherungspolitik präferenzfrei, wenn

$$
E_{t-2}\left(\varepsilon_{t-1} \mid \pi_{t}\left(\varepsilon_{t-1}\right)=\pi_{t}\right)=0 \quad \forall \quad \pi_{t} .
$$

Dies folgt, weil bei einem Devisenterminkontrakt $\ni_{t-1}=\varepsilon_{t-1}$ gilt. Bedingung (15) ist z.B. erfüllt, wenn Proposition 4.3 gilt.

Proposition 4.3. Das Exportergebnis $\pi^{P}\left(e_{t-1}\right)$ weise konstante Konvexität auf und $\varepsilon_{t-1}$ gehorche einer um 0 symmetrischen Wahrscheinlichkeitsverteilung. Dann ist die optimale präferenzfreie Absicherung gegeben durch

$$
Z_{t-2} r_{t-1, t}=\left.\frac{\partial \pi^{p}\left(e_{t-1}\right)}{\partial e_{t-1}}\right|_{e_{t-1}=E_{t-2}\left(e_{t-1}\right)}
$$

Beweis. Die Absicherungspolitik (16) bedeutet eine deltaneutrale Absicherung des Exportergebnisses an der Stelle des erwarteten Wechselkurses $E_{t-2}\left(e_{t-1}\right)$. Diese Politik hat zur Folge, dass gemäß Gleichung (13) das Gesamtergebnis eine nach oben geöffnete Parabel ist, die ihren Scheitel bei $e_{t-1}=E_{t-2}\left(e_{t-1}\right)$ hat.

Folglich ist

$$
\pi_{t}\left(E_{t-2}\left(e_{t-1}\right)+\varepsilon_{t-1}\right)=\pi_{t}\left(E_{t-2}\left(e_{t-1}\right)-\varepsilon_{t-1}\right) ; \quad \forall \quad \varepsilon_{t-1} .
$$

Dann ist Bedingung (15) erfüllt, wenn $\forall \varepsilon_{t-1}$ die Wahrscheinlichkeit(sdichte) von $+\varepsilon_{t-1}$ mit der von $-\varepsilon_{t-1}$ übereinstimmt. Dies ist bei symmetrischer Wahrscheinlichkeitsverteilung der Fall

Eine deltaneutrale Absicherung des Exportergebnisses an der Stelle des erwarteten Wechselkurses kann interpretiert werden als eine lokale Vollabsicherung, bezogen auf diesen Wechselkurs. Hiermit wird das Wechselkursrisiko minimiert. Eine spekulative Position entfällt auch im Zeitpunkt $(t-2)$, da keine Risikoprämie gezahlt wird.

Die deltaneutrale Absicherung ist optimal, wenn Bedingung (17) gilt und $\varepsilon_{t-1}$ symmetrisch verteilt ist. Bedingung (17) ist bei deltaneutraler Absicherung auch erfüllt, wenn für das Exportergebnis gilt

$$
\pi^{p}\left(E_{t-2}\left(e_{t-1}\right)+\varepsilon_{t-1}\right)-\pi^{p}\left(E_{t-2}\left(e_{t-1}\right)\right)=\pi^{p}\left(E_{t-2}\left(e_{t-1}\right)\right)-\pi^{p}\left(E_{t-2}\left(e_{t-1}\right)-\varepsilon_{t-1}\right) ; \quad \forall \varepsilon_{t-1}(18
$$

Gemäß dieser Bedingung weicht das Exportergebnis betragsmäßig von demjenigen beim erwarteten Wechselkurs gleichermaßen bei $+\varepsilon_{t-1}$ und $-\varepsilon_{t-1}$ ab. Insofern besteht Symmetrie um den erwarteten Wechselkurs. Diese und eine symmetrische Verteilung von $\varepsilon_{t-1}$ genügen, um die Optimalität einer deltaneutralen Absicherung zu gewährleisten. Die Problematik der Bedingung resultiert jedoch daraus, dass sie nicht gleichzeitig für verschiedene erwartete Wechselkurse $E_{t-2}\left(e_{t-1}\right)$ gilt, es sei denn, dass $\pi^{P}\left(e_{t-1}\right)$ konstante Konvexität aufweist. 
Im folgenden untersuchen wir die Absicherungspolitik in den vorangehenden Perioden und unterstellen einerseits konstante Konvexität von $\pi^{P}\left(e_{t-1}\right)$ und andererseits, dass in jeder Periode $\tau$ die Wechselkursinnovation $\varepsilon_{\tau}$ symmetrisch um 0 verteilt ist. Dann lässt sich die optimale Absicherungspolitik in jeder Periode $\tau$ charakterisieren. $r_{\tau, t}$ sei der risikofreie Aufzinsungsfaktor zwischen den Zeitpunkten $\tau$ und $t$.

Proposition 4.4. Das Exportergebnis $\pi^{P}\left(e_{t-1}\right)$ sei eine Funktion mit konstanter Konvexität; die Wechselkursinnovation $\varepsilon_{\tau}$ sei in jeder Periode symmetrisch um 0 verteilt. Dann ist es für die Firma optimal, im Zeitpunkt $\tau Z_{\tau}$ einperiodige Devisenterminkontrakte zum Kurs $f_{\tau}$ zu verkaufen mit

$$
Z_{\tau} r_{\tau+1, t}=\left.\frac{\partial \pi^{p}\left(e_{t-1}\right)}{\partial e_{t-1}}\right|_{e_{t-1}=E_{\tau}\left(e_{t-1}\right)} ; \quad \tau=0, \ldots, t-2 .
$$

Beweis. Zunächst wird der Beweis für die Absicherungspolitik im Zeitpunkt $(t-2)$ erbracht. Das Gesamtergebnis im Zeitpunkt $t$ ist bei Einbezug der Absicherungspolitik in den Zeitpunkten $0, \ldots, t-2$ gleich

$$
\begin{aligned}
\pi_{t} & =\pi^{p}\left(e_{t-1}\right)+\sum_{\tau=0}^{t-2} Z_{\tau}\left(f_{\tau}-e_{\tau+1}\right) r_{\tau+1, t} \\
& =\pi^{p}\left(e_{t-1}\right)-\sum_{\tau=0}^{t-2} Z_{\tau} \varepsilon_{\tau+1} r_{\tau+1, t}
\end{aligned}
$$

Sei

$$
H_{t-\alpha}=-\sum_{\tau=0}^{t-\alpha-1} Z_{\tau} \varepsilon_{\tau+1} r_{\tau+1, t}
$$

das Absicherungsergebnis aus den Perioden vor dem Zeitpunkt $(t-\alpha)$. Im Zeitpunkt $(t-\alpha)$ ist dieses bekannt. Daher beeinflußt es beide Seiten von (17) im Zeitpunkt $(t-2)$ gleichermaßen, so dass im Zeitpunkt $(t-2)$ die bereits diskutierte deltaneutrale Absicherungspolitik optimal ist. Diese Politik bewirkt, ausgehend von $e_{t-2}$, dass $\pi_{t}\left(e_{t-1}\right)$ eine nach oben geöffnete Parabel ist mit dem Scheitelpunkt bei $e_{t-1}=E_{t-2}\left(e_{t-1}\right)$.

Jetzt wird die Absicherung im Zeitpunkt $(t-3)$ analysiert, ausgehend von der Politik $Z_{t-2}^{*}\left(e_{t-2}\right) . Z_{t-3}$ ist optimal, wenn gilt

$$
E_{t-3}\left[u^{\prime}\left(\pi_{t}\right) \varepsilon_{t-2}\right]=0
$$

Aus Gleichung (1) folgt

$$
e_{t-1}=\gamma^{2} e_{t-3}+\left(1-\gamma^{2}\right) \bar{e}+\gamma \varepsilon_{t-2}+\varepsilon_{t-1} .
$$

$\varepsilon_{t-1}$ ist unabhängig von $\varepsilon_{t-2} \cdot \pi_{t}=\pi_{t}\left(\varepsilon_{t-2}, \varepsilon_{t-1}\right)$ wegen (22).

Da $\varepsilon_{t-2}$ und $\varepsilon_{t-1}$ symmetrisch verteilt sind, ist Bedingung (21) erfüllt, wenn

$$
\pi_{t}\left(\varepsilon_{t-2}, \varepsilon_{t-1}\right)=\pi_{t}\left(-\varepsilon_{t-2}, \varepsilon_{t-1}\right)=\pi_{t}\left(\varepsilon_{t-2},-\varepsilon_{t-1}\right)=\pi_{t}\left(-\varepsilon_{t-2},-\varepsilon_{t-1}\right) ; \quad \forall \quad\left(\varepsilon_{t-2}, \varepsilon_{t-1}\right)
$$

gilt. Zunächst sei $Z_{t-2}=0$ unterstellt. Da $\pi_{t}^{P}\left(\varepsilon_{t-1}\right)$ konstante Konvexität aufweist, bewirkt eine deltaneutrale Absicherung $Z_{t-3}$, dass

$$
\pi_{t}\left(\varepsilon_{t-2}, \varepsilon_{t-1}\right)=\pi_{t}\left(-\varepsilon_{t-2},-\varepsilon_{t-1}\right) ; \quad \forall \quad\left(\varepsilon_{t-2}, \varepsilon_{t-1}\right) .
$$


$\pi_{t}$ ist eine nach oben geöffnete Parabel, die ihr Minimum an der Stelle $\varepsilon_{t-2}=\varepsilon_{t-1}=0$ aufweist. Bedingung (21) ist somit erfüllt. Wenn nun im Zeitpunkt $(t-2)$ eine optimale Absicherungspolitik $Z_{t-2}^{*}\left(e_{t-2}\right)=Z_{t-2}^{*}\left(\varepsilon_{t-2}\right)$ hinzugefügt wird, dann bewirkt diese, dass $\forall \varepsilon_{t-2}$

$$
\pi_{t}\left(\varepsilon_{t-2}, \varepsilon_{t-1}\right)=\pi_{t}\left(\varepsilon_{t-2},-\varepsilon_{t-1}\right) ; \quad \forall \quad \varepsilon_{t-1} .
$$

Da aufgrund der deltaneutralen Politik $Z_{t-3}^{*}$ auch (24) gilt, ist Bedingung (23) erfüllt. Analog verläuft der Beweis für die vorangehenden Zeitpunkte

Proposition 4.4 wird in Abb. 1 veranschaulicht. Das Exportergebnis $\pi^{P}\left(e_{t-1}\right)$ ist eine ansteigende Kurve mit konstanter Konvexität. Durch Verkauf von $Z_{t-3}^{*}$ Devisenterminkontrakten im Zeitpunkt $(t-3)$ erzielt die Firma ein deltaneutrales Gesamtergebnis an der Stelle $E_{t-3}\left(e_{t-1}\right)$. An dieser Stelle hat die Parabel $\pi_{t}\left(e_{t-1} \mid Z_{t-3}\right)$ ihr Minimum.

Nach einer Periode, also im Zeitpunkt $(t-2)$, ändere sich der erwartete Wechselkurs des Zeitpunktes $(t-1)$ um $\gamma \varepsilon_{t-2}^{0}$ bzw. $-\gamma \varepsilon_{t-2}^{0}$. Folglich ist die Position der Firma nicht mehr deltaneutral in bezug auf den neuen Erwartungswert $E_{t-2}\left(e_{t-1}\right)$. Dies schlägt sich in der Zahl der Devisenterminkontrakte nieder, die die Firma im Zeitpunkt $(t-2)$ verkauft. Wäre $E_{t-3}\left(e_{t-1}\right)=E_{t-2}\left(e_{t-1}\right)$, dann würde sich die Zahl der Terminkontrakte im Zeitablauf nur ändern, weil der Aufzinsungsfaktor $r_{\tau, t}$ kleiner wird und der Faktor $\gamma^{t-\tau-1}$ wächst. Dieser Faktor resultiert aus dem OrnsteinUhlenbeck-Prozess, wonach gilt

$$
\frac{\partial E_{\tau}\left(e_{t-1}\right)}{\partial e_{\tau}}=\gamma^{t-\tau-1}
$$

Das Gesamtergebnis einschließlich der Absicherung im Zeitpunkt $(t-2)$ ist wiederum eine nach oben geöffnete Parabel, die ihr Minimum nun an der Stelle des neuen Erwartungswertes $E_{t-2}\left(e_{t-1}\right)$ annimmt.

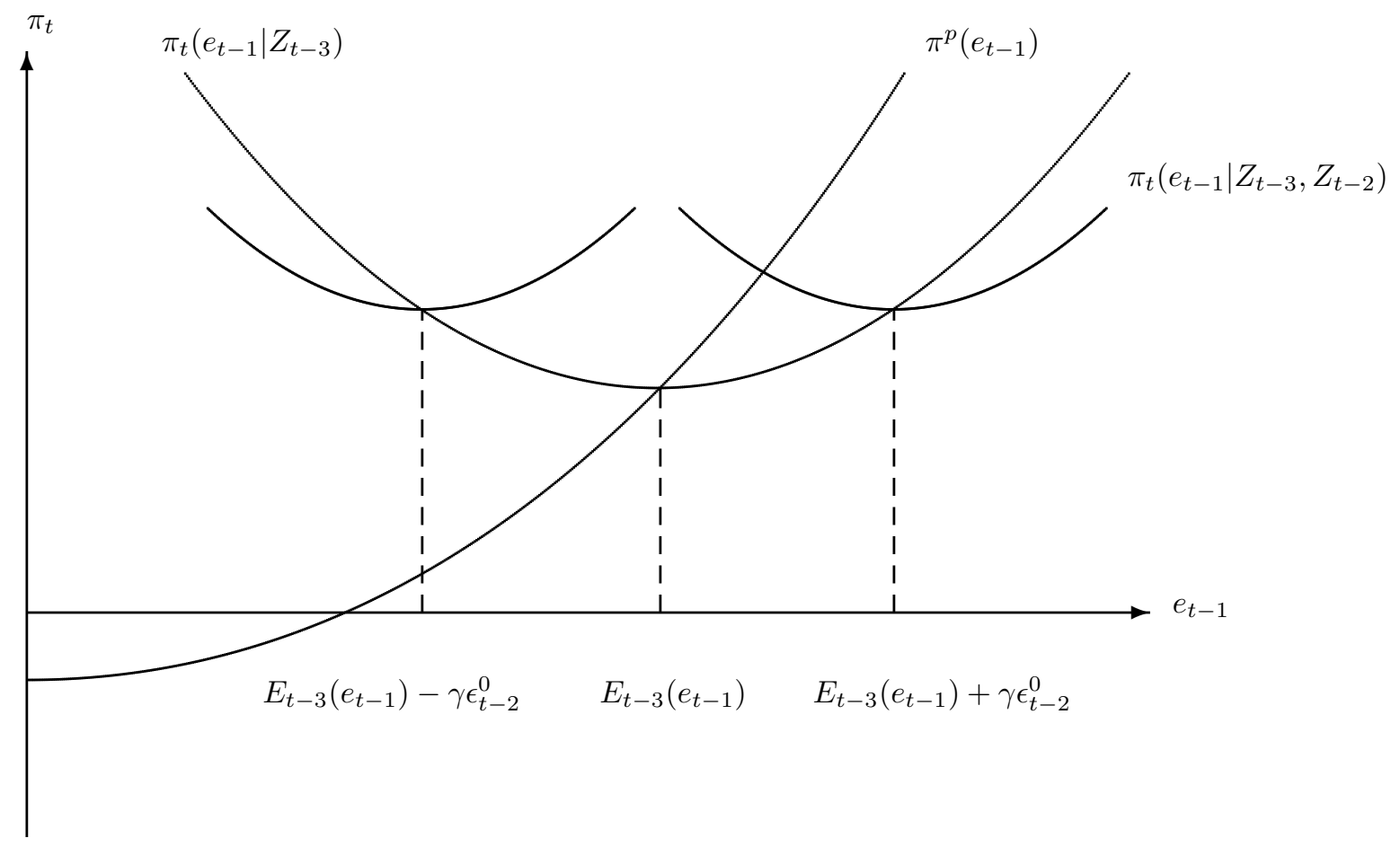

Abbildung 1: Sie zeigt das ansteigende, konvexe Exportergebnis $\pi^{P}\left(e_{t-1}\right)$, ebenso das Gesamtergebnis $\pi_{t}\left(e_{t-1} \mid Z_{t-3}\right)$ ohne Absicherung im Zeitpunkt $(t-2)$ und schließlich das Gesamtergebnis 
$\pi_{t}\left(e_{t-1} \mid Z_{t-3}, Z_{t-2}\right)$ unter Einschluss der Absicherung im Zeitpunkt $(t-2)$; bedingt auf $\pm \gamma \varepsilon_{t-2}^{0}$.

Diese einfache Absicherungspolitik wird von folgenden Überlegungen gestützt. (1) Da es keine Risikoprämie im Devisenmarkt gibt, entfällt eine spekulative Position im Terminmarkt. (2) Konstante Konvexität des Exportergebnisses und symmetrische Verteilung der voneinander unabhängigen Wechselkursinnovationen erzeugen ein "voll symmetrisches" Absicherungsproblem. Daher gibt es keinen Anreiz für eine Teil- oder Übersicherung in der Mitte, d.h. an der Stelle des erwarteten Wechselkurses $E_{\tau}\left(e_{t-1}\right)$. (3) Diese Modellstruktur erzeugt keine Stochastik des zukünftigen Entscheidungsspielraums, gegen die sich die Firma ebenfalls absichern möchte. Daher ist die Absicherungspolitik besonders einfach. Sie ist jedoch nicht untypisch. Denn die deltaneutrale Absicherungspolitik in Periode $\tau$ zielt nicht auf den erwarteten Wechselkurs am Periodenende, sondern auf den im Zeitpunkt $(t-1)$.

Dennoch muss die Firma ein Restwechselkursrisiko tagen, weil sie nur Devisenterminkontrakte einsetzen kann. Dieses Risiko würde in einem zeitstetigen Modell mit einem Ornstein-UhlenbeckDiffusionsprozess verschwinden. Dann gäbe es einen dynamisch vollständigen Devisenmarkt, so dass alle Wechselkursrisiken abgesichert werden könnten.

\section{Diskussion und Zusammenfassung}

Die in Proposition 4.4 angegebene Absicherungspolitik hat verschiedene Eigenschaften.

Die Absicherungspolitik ist einfach. Komplexe intertemporale Strategien erübrigen sich.

Um diese Politik zu implementieren, muss die Firma die Exportergebnisfunktion $\pi^{P}\left(e_{t-1}\right)$ und den mean reversion-Parameter $\gamma$ kennen. Die Verteilungen der Wechselkursinnovationen muss sie nicht kennen. Allerdings müssen diese symmetrisch sein.

Die deltaneutrale Absicherungspolitik ist präferenzfrei. Sie gilt für alle risikoaversen Firmen. Auch Belastungen, die vom Gesamtergebnis abhängen, wie z.B. Gewinnsteuern oder Kosten finanzieller Anpassung, verändern das Resultat nicht.

Bisher wurde nur der Export in Periode $t$ betrachtet. Dieselben Überlegungen gelten, wenn in jeder Periode exportiert wird. Die optimale Exportpolitik in Periode $\tau$ wird ebenfalls anhand des Separationstheorems bestimmt. Wichtig ist, dass die Exportergebnisfunktion $\pi^{P}\left(e_{\tau-1}\right)$ ebenfalls konstante Konvexität aufweist. Dann kann für jede Exportperiode das Wechselkursrisiko gemäß Proposition 4.4 abgesichert werden. Die Gesamtzahl der zu verkaufenden Devisenterminkontrakte ist dann gleich der Summe der periodenbezogenen Kontrakte. Die Absicherungspolitik bleibt daher dieselbe.

Häufig exportiert eine Firma mehrere Produktarten. Wichtig ist nun, dass das über alle Produktarten aggregierte Exportergebnis $\pi^{P}\left(e_{t-1}\right)$ konstante Konvexität aufweist. Dann bleibt die in Proposition 4.4 angegebene Absicherungspolitik optimal.

Möglicherweise entscheidet die Firma über den Export erst, wenn sie den Wechselkurs, zu dem der Exporterlös abgerechnet wird, schon kennt. Dann ist die Exportentscheidung risikolos. Das Exportergebnis in Periode $t$ ist dann $\pi_{t}^{P}\left(e_{t}\right)$. Folglich sichert die Firma dieses Ergebnis nun auch in Periode $t \mathrm{ab}$, nicht nur in den vorangehenden Perioden. Wenn $\pi_{t}^{P}\left(e_{t}\right)$ konstante Konvexität aufweist, ist es nach wie vor optimal, eine deltaneutrale Absicherungspolitik zu wählen, jetzt allerdings bezogen auf den Erwartungswert $E_{\tau}\left(e_{t}\right)$ anstelle von $E_{\tau}\left(e_{t-1}\right)$.

Schließlich erlauben diese Ergebnisse auch einige Schlussfolgerungen zu der Kontroverse zwischen Risikoaversionshypothese und Exportoptionshypothese. Die Ergebnisse stützen die zweite Hypothese. Wie Abbildung 1 zeigt, begünstigt eine Zunahme der Wechselkursvolatilität die Firma. Die Firma nehme ihre Aktivitäten im Zeitpunkt $(t-3)$ auf. Wenn die Wechselkursvolatilität gering ist, liegt das Gesamtergebnis nahe beim Minimum von $\pi_{t}\left(e_{t-1} \mid Z_{t-3}\right)$. Je höher $\left|\varepsilon_{t-2}^{0}\right|$ ist, um so höher ist das Gesamtergebnis. Eine Zunahme der Wechselkursvolatilität erzeugt daher eine Verbesserung im Sinn stochastischer Dominanz 1. Grades.

Ähnlich wirkt eine Zunahme der Wechselkursvolatilität in Periode $(t-1)$. Je größer $\left|\varepsilon_{t-1}\right|$ ist, um so höher ist das Gesamtergebnis $\pi_{t}\left(e_{t-2} ; e_{t-1} \mid Z_{t-3}, Z_{t-2}\right)$ bei deltaneutraler Absicherung in 
den Zeitpunkten $(t-3)$ und $(t-2)$. Wiederum kommt es zu einer Verbesserung im Sinn stochastischer Dominanz 1. Grades. Die Risikoaversionshypothese greift nicht, weil das Exportrisiko in der Exportperiode gemäß dem Separationstheorem vollständig abgesichert werden kann. Wenn weitere nicht absicherbare Risiken bestehen, dann greift die Risikoaversionshypothese. Die Risikoaversion spielt dann eine Rolle für die Exportentscheidung. Dies impliziert nicht, dass Wechselkursvolatilität die Firma benachteiligt. Denn die Exportoption wächst i.a. im Wert mit der Wechselkursvolatilität. Allerdings ist zu fragen, ob bei einer Zunahme der Wechselkursvolatilität die Exportergebnisfunktion $\pi_{t}^{P}\left(e_{t-1}\right)$ unverändert bleibt. Wenn eine höhere Volatilität neue Wettbewerber zum Markteintritt oder vorhandene zum Marktaustritt bewegt, dann ändert sich die Wettbewerbsintensität und damit die Exportergebnisfunktion. Daher sind die Ergebnisse vorsichtig im Sinn einer komparativ statischen Analyse zu interpretieren, nicht im Sinn einer Gleichgewichtsanalyse, die auch den Zusammenhang zwischen Wechselkursvolatilität und Wettbewerbsintensität einbezieht. 


\section{Literaturverzeichnis}

[1] Adam-Müller, A. (1995), "Internationale Unternehmensaktivität, Wechselkursrisiko und Hedging mit Finanzinstrumenten", Heidelberg: Physica Verlag.

[2] Adam-Müller, A. (2002), "What to Do If a Dollar is not a Dollar? The Impact of Inflation Risk on Production and Risk Management", Journal of Futures Markets, 22, 371-386.

[3] Adam, T. (2002), "Why Firms Use Non-Linear Hedging Strategies", Discussion Paper, Hong Kong University of Science and Technology.

[4] Allayannis, G. und Ofek, E. (2001), "Exchange Rate Exposure, Hedging, and the Use of Foreign Currency Derivatives", Journal of International Money and Finance, 20, 273-296.

[5] Benninga, S., Eldor, R. und Zilcha I. (1985), "Optimal International Hedging in Commodity and Currency Forward Markets", Journal of International Money and Finance, 4, 537-552.

[6] Bodnar, G.M., Dumas, B. und Marston, R.C. (2002), "Pass-Through and Exposure", Journal of Finance, 57, 199-231.

[7] Breuer, W. (2000), "Unternehmerisches Währungsmanagement", 2.Auflage, Gabler, Wiesbaden.

[8] Broll, U. und Wahl, J. (1992), "International Investments and Exchange Rate Risk", European Journal of Political Economy, 8, 31-40.

[9] Broll, U., Wahl, J. und Zilcha, I. (1999), "Hedging Exchange Rate Risk: The Multiperiod Case", Research in Economics, 53, 365-380.

[10] Dixit, A. und Stiglitz J.E. (1977), "Monopolistic Competition and Optimum Product Diversity", American Economic Review, 67, 297-308.

[11] Engle, C. (1996), "The Forward Discount Anomaly and the Risk Premium: A Survey of Recent Evidence", Journal of Empirical Finance, 3, 123-191.

[12] Franke, G. (1991), "Exchange Rate Volatility and International Trading Strategy", Journal of International Money and Finance, 10, 292-307.

[13] Franke, G. (2003), "Changing Horses and Hedging", in Rauscher, M., Pethig, R., ed., Challenges to the World Economy, Berlin: Springer, 261-275.

[14] Froot, K., Scharfstein, D. und Stein J. (1993), "Risk Management: Coordinating Investment and Financing Decisions", Journal of Finance, 48, 1629-1658.

[15] Guay, W. und Kothari, S.P. (2003), "How Much Do Firms Hedge with Derivatives?", Journal of Financial Economics, 70, 423-461.

[16] Gollier, C. (1995), "The comparative statics of changes in risk revisited", Journal of Economic Theory $66,522-536$.

[17] Haushalter, G.D. (2000), "Financing Policy, Basis Risk, and Currency Hedging: Evidence from Oil and Gas Producers", Journal of Finance, 55, 107-152.

[18] Holthausen, D. (1979), "Hedging and the Competitive Firm Under Price Uncertainty", American Economic Review 69, 989-995.

[19] Kawai, M. und Zilcha, I. (1986), "International Trade with Forward-Futures Markets under Exchange Rate and Price Uncertainty", Journal of International Economics, 20, 83-98.

[20] Mello, A., Parsons, J. und Triantis, A.J. (1995), "An Integrated Model of Multinational Flexibility and Financial Hedging", Journal of International Economics, 39, 27-51.

[21] Neuberger, A. (1999), "Hedging Long-Term Exposure with Multiple Short-Term Futures Contracts", Review of Financial Studies, 12, 429-459.

[22] Sercu, P. und VanHulle, C. (1992), "Exchange Rate Volatility, International Trade and the Value of Exporting Firms", Journal of Banking and Finance, 16, 155-182.

[23] Ungern-Sternberg, V. und Von Weizsäcker, C. (1990), "Strategic Foreign Exchange Management", Journal of Industrial Economics, 38, 381-395.

[24] Ware, R. und Winter, R. (1988), "Forward Markets, Currency Options and the Hedging for Foreign Exchange Risk", Journal of International Economics, 291-302.

[25] Wilhelm, J. (1992), "Fristigkeitsstruktur und Zinsänderungsrisiko - Vorüberlegungen zu einer Markowitz-Theorie des Bond-Portfolio-Management", Zeitschrift für betriebswirtschaftliche Forschung, 44, 209-246.

[26] Wilhelm, J. (1999),"A Fresh View on the Ho-Lee Model of the Term Structure From a Stochastic Discounting Perspective", in: Kürsten, W. und Wilhelm, J. (Hrsg.): "OR Spektrum Special Issue Finance and Banking", OR Spektrum 21,9-34. 